\title{
P-63
}

\section{Cholinergic and Antioxidant Effects of a Novel Neuroprotective Compound HAB16R26 Exposed in Sprague Dawley Rats}

\author{
Siti Norshazwani Wahab ${ }^{\mathrm{a}}$, Kalavathy Ramasamy ${ }^{\mathrm{b}}$, Abu Bakar Abdul Majeed ${ }^{\mathrm{a}}$ and Vasudevan \\ Mani $^{\mathrm{a}, *}$ \\ ${ }^{a}$ Brain Research Laboratory, Faculty of Pharmacy, UiTM Puncak Alam, 42300 Bandar Puncak Alam, Selangor Darul \\ Ehsan, Malaysia; ${ }^{b}$ Collaborative Drug Development and Research (CDDR) Group, Faculty of Pharmacy, UiTM Puncak \\ Alam, 42300 Bandar Puncak Alam, Selangor Darul Ehsan, Malaysia; E-mail: vasudevan@puncakalam.uitm.edu.my
}

Endophytes, the microorganisms that live inside other living organisms believed to preserve a lot of potential bioactivity compounds. A novel neuroprotective compound isolated from an endophyte, HAB16R26; Cytospora rhizophorae, was newly discovered. It able to inhibit beta-secretase (BACE1) (IC50BACE1 $=2.2 \mu \mathrm{g} / \mathrm{ml})$. Through the amyloidogenic pathway, BACE1 enzyme responsible in synthesizing amyloid precursor protein (APP) to beta amyloid (A $\beta$ ) which then accumulates as A $\beta$ plaques, one of the hallmark in brain of Alzheimer's disease (AD) patients. The present study aimed to investigate the potential effect of a neuroprotective compound isolated from endophyte, HAB16R26 on cholinergic and antioxidant systems. Male and female Sprague Dawley (SD) rats $(\mathrm{n}=5)$ were treated with two different concentrations ( 2 and $5 \mathrm{mg} / \mathrm{kg}$ ) of compound for 28 days via oral gavage. At the end of the treatment, all the animals were sacrificed. The brains were collected for cholinergic and antioxidant assays. Interestingly, in male treated group, the level of acetylcholine (ACh) significantly increased as compared to control group meanwhile acetylcholinesterase (AChE) level of female treated group significantly inhibited when compared to control group. Level production of nitric oxide (NO) was significantly inhibited in both treated groups as compared to control group. Activity of catalase (CAT) enzyme found to be significant inhibited in female treated group compared to control group. However there were none significant differences of superoxide dismutase (SOD) and glutathione (GSH) activity levels observed. Results showed the compound have potential in enhancing the brain cholinergic system and several antioxidant mechanisms.

Keywords: Alzheimer's disease, antioxidant, cholinergic, endophytes, neuroprotective. 